\title{
Holography and the Weyl anomaly
}

\author{
Måns Henningson ${ }^{1}$ and Kostas Skenderis ${ }^{2}$ \\ 1 Institute of Theoretical Physics, \\ Chalmers University of Technology, \\ S-412 96 Göteborg, Sweden \\ mans@fy. chalmers.se \\ 2 Spinoza Institute, University of Utrecht, \\ Leuvenlaan 4, 3584 CE Utrecht, The Netherlands \\ k.skenderis@phys.uu.nl
}

\begin{abstract}
We review our calculation of the Weyl anomaly for $d$-dimensional conformal field theories that have a description in terms of a $(d+1)$-dimensional gravity theory.
\end{abstract}

\section{Introduction}

In the last few months, we have learned that a theory containing gravity and defined on an open $(d+1)$-manifold $X$ can, in some cases, be equivalent to a $d$-dimensional conformal field theory defined on the boundary $M$ of $X$ [1], [2], [3]. The manifold $X$ has a causal structure similar to that of $(d+1)$-dimensional anti-de Sitter space, i.e. the boundary $M$ at conformal infinity is timelike. This means that the space $X$ is not globally hyperbolic, i.e. it does not admit any Cauchy surfaces, since new information may come in through the boundary $M$. The action functional $S[\phi]$ or the equations of motion for the gravity theory on $X$ therefore do not completely determine the dynamics and must be supplemented by appropriate boundary data $\phi_{(0)}$ on $M$ for each field $\phi$ on $X$. The partition function is then a functional of these boundary data:

$$
Z_{\text {grav }}\left[\phi_{(0)}\right]=\int_{\phi_{(0)}} \mathcal{D} \phi \exp (-S[\phi]),
$$

where the subscript on the integration sign indicates that the functional integral is over field configurations $\phi$ that satisfy the boundary condition given by $\phi_{(0)}$. The precise relationship between this gravity theory on $X$ and the conformal field theory on the boundary $M$ can now be described as follows: There is a oneto-one correspondence between the fields $\phi$ on $X$ and the primary operators $\mathcal{O}$ on $M$. The set of correlation functions of the latter are conveniently summarized by a generating functional:

$$
Z_{C F T}\left[\phi_{(0)}\right]=\left\langle\exp \int_{M} d^{d} x \mathcal{O} \phi_{(0)}\right\rangle,
$$

where $\phi_{(0)}$ is now regarded as a formal expansion parameter. The partition function of the gravity theory on $X$ and the generating functional on $M$ are 
then equal, regarded as functionals of $\phi_{(0)}$ :

$$
Z_{\text {grav }}\left[\phi_{(0)}\right]=Z_{C F T}\left[\phi_{(0)}\right] .
$$

This relationship is particularly useful when the gravity theory is weakly coupled. We can then calculate the partition function at tree level, where it is given by the exponential of the action functional evaluated for a field configuration $\phi^{c l}\left(\phi_{(0)}\right)$ that solves the classical equations of motion and obeys the boundary conditions given by $\phi_{(0)}$ :

$$
Z_{\text {grav }}^{\text {tree }}\left[\phi_{(0)}\right]=\exp \left(-S\left[\phi^{c l}\left(\phi_{(0)}\right)\right]\right) .
$$

A field of particular importance in a theory containing gravity is of course the metric $G_{\mu \nu}$. The corresponding operator in the boundary conformal field theory is the stress-energy tensor $T_{i j}$. The boundary data for the metric $G_{\mu \nu}$ is not a boundary metric $g_{(0)} i j$, but only a conformal structure $\left[g_{(0)}\right]$. (This is defined as an equivalence class of boundary metrics when two metrics that differ by a local rescaling are considered equivalent, i.e. $g_{(0)} \sim \exp 2 \sigma(x) g_{(0)}$ for an arbitrary positive function $\sigma(x)$.) Indeed, the metric $G_{\mu \nu}$ on $X$ has a second order pole at the boundary $M$ and thus does not induce a metric there. To get a finite metric on $M$, we could multiply $G_{\mu \nu}$ by $f^{2}$, where $f$ is some positive function on $X$ with a simple zero on $M$ and then restrict to $M$. The freedom to choose $f$ means that only the conformal equivalence class of the metric so obtained is unambiguously defined. Conversely, a choice of conformal structure $\left[g_{(0)}\right]$ on $M$ together with Einstein's equations on $X$ suffice to determine an (up to diffeomorphisms) unique metric $G_{\mu \nu}$ on $X$ [4]. We thus naturally get a conformal field theory on $M$. Indeed, to consider a more general theory on $M$, we would need a background metric. However, as we have seen, only the conformal structure of $M$ is well-defined, so only conformal field theories are meaningful on $M$.

In the following, we will consider the effective action $W_{C F T}\left[g_{(0)}\right]=-\log Z_{C F T}\left[g_{(0)}\right]$ of the theory on $M$. This can be regarded as minus the logarithm of the zeropoint function or partition function of the theory. (It is a zero-point function in the sense that there are no insertions of any operators). A priori, this is a functional of the metric $g_{(0)}$ on $M$, but by conformal invariance, it should actually only depend on the conformal equivalence class $\left[g_{(0)}\right]$. However, this invariance is sometimes broken by a conformal (or Weyl) anomaly. This means that $W_{C F T}\left[g_{(0)}\right]$ is not invariant under a conformal rescaling $\delta g_{(0)}=2 \delta \sigma g_{(0)}$ of the metric, but transforms as

$$
\delta W_{C F T}\left[g_{(0)}\right]=\int_{M} d^{d} x \sqrt{\operatorname{det} g_{(0)}} \mathcal{A} \delta \sigma,
$$

where $\mathcal{A}$ is the anomaly. On general grounds, the gravitational part of the Weyl anomaly vanishes when the dimension $d$ of $M$ is odd. When $d$ is even, it is of the form

$$
\mathcal{A}=E+I+D_{i} J^{i}
$$


where $E$ is proportional to the $d$-dimensional Euler density, and $I$ is a conformal invariant [5] [6]. The total derivative term $D_{i} J^{i}$ is irrelevant, since it can be canceled by adding a local counterterm to the effective action. There is of course a unique Euler density in every even dimension $d$. The number of linearly independent conformal invariants grows with $d$, though. There are no such invariants for $d=2$, one (the square of the Weyl tensor) for $d=4$, three for $d=6$, etcetera. We will outline how to calculate the conformal anomaly for $d$-dimensional conformal field theories that have a description in terms of a $(d+1)$-dimensional gravity theory as described above. Technical details (and some intermediate results not presented in [7]) are relegated to the appendix.

\section{The calculation}

According to the recipe above, $W_{C F T}\left[g_{(0)}\right]$ is given by minus the logarithm of the partition function of the gravity theory with a certain conformal structure $\left[g_{(0)}\right]$ induced by the metric $G_{\mu \nu}$ on the boundary. All other fields of the gravity theory should vanish on the boundary $M$, since we are not inserting any operators in the conformal field theory correlation function. If a tree-level computation is justified, so that we only need to consider field configurations on $X$ that solve the classical equations of motion, this means that all fields expect the metric vanish everywhere on $X$. The theory in bulk is then reduced to pure gravity described by the Einstein-Hilbert action plus a cosmological constant term:

$$
S_{\text {bulk }}=\frac{1}{16 \pi G_{N}} \int_{X} d^{d+1} x \sqrt{\operatorname{det} G_{\mu \nu}}(R+2 \Lambda) .
$$

On a manifold with boundary, we also have the term

$$
S_{\text {boundary }}=\frac{1}{16 \pi G_{N}} \int_{M} d^{d} x \sqrt{\operatorname{det} \tilde{g}_{i j}} 2 K,
$$

where $\tilde{g}_{i j}$ is the metric on the boundary and $K$ is the trace of the second fundamental form. This boundary term is necessary in order to get an action that depends only on first derivatives of the metric[8]. As described in the first section, a choice of conformal structure $\left[g_{(0)}\right]$ on the boundary $M$ determines a unique metric $G_{\mu \nu}$ in the bulk of $X$ that solves the equations of motion

$$
R_{\mu \nu}-\frac{1}{2} R G_{\mu \nu}=\Lambda G_{\mu \nu}
$$

(This is of course true only up to diffeomorphisms). However, the bulk action diverges when evaluated for such a field configuration because of the second order pole in $G_{\mu \nu}$ on the boundary. Furthermore, the boundary terms in the action are ill-defined, since $G_{\mu \nu}$ does not induce a finite metric $\tilde{g}_{i j}$ on $M$.

To regulate the theory in a manner consistent with general covariance, we pick a specific representative $g_{(0)}$ of the boundary conformal structure $\left[g_{(0)}\right]$. 
This determines a distinguished coordinate system $\left(\rho, x^{i}\right)$, in which the metric on $X$ takes the form [9]

$$
G_{\mu \nu} d x^{\mu} d x^{\nu}=\frac{l^{2}}{4} \rho^{-2} d \rho d \rho+\rho^{-1} g_{i j} d x^{i} d x^{j} .
$$

Here the tensor $g$ has the limit $g_{(0)}$ as one approaches the boundary represented by $\rho=0$. The length scale $l$ is related to the cosmological constant $\Lambda$. Einstein's equations for $G_{\mu \nu}$ can then be solved order by order in $\rho$ with the result that $g$ is of the form

$$
g=g_{(0)}+\rho g_{(2)}+\ldots+\rho^{d / 2} g_{(d)}+\rho^{d / 2} \log \rho h_{(d)}+\mathcal{O}\left(\rho^{d / 2+1}\right) .
$$

The regularization procedure now amounts to restricting the bulk integral in the action to the domain $\rho>\epsilon$ for some cutoff $\epsilon>0$ and evaluating the boundary integrals at $\rho=\epsilon$. The regulated action evaluated for this field configuration is then of the form

$$
\begin{aligned}
W\left[g_{(0)}\right]= & \frac{1}{16 \pi G_{N}} \int d^{d} x \sqrt{\operatorname{det} g_{(0)}}\left(\epsilon^{-d / 2} a_{(0)}+\epsilon^{-d / 2+1} a_{(2)}+\ldots+\epsilon^{-1} a_{(d-2)}-\log \epsilon a_{(d)}\right) \\
& +W_{f i n}\left[g_{(0)}\right] .
\end{aligned}
$$

The coefficients $a_{(0)}, a_{(2)}, \ldots, a_{(d)}$ are all given by covariant expressions in $g_{(0)}$ and its curvature tensor $R_{j k l}^{i}$ (see appendix). The divergences as $\epsilon$ goes to zero can thus be canceled by adding local counterterms to the action, so that we are left with a finite effective action $W_{f i n}\left[g_{(0)}\right]$. To find the variation of $W_{f i n}\left[g_{(0)}\right]$ under a conformal rescaling of the boundary metric $g_{(0)}$, we note that the regulated action $W\left[g_{(0)}\right]$ is invariant under the combined transformation $\delta g_{(0)}=2 \delta \sigma g_{(0)}$ and $\delta \epsilon=2 \delta \sigma \epsilon$ for a constant parameter $\delta \sigma$. The terms proportional to negative powers of $\epsilon$ are separately invariant, so the variation of $W_{f i n}\left[g_{(0)}\right]$ must therefore equal minus the variation of the logarithmically divergent term. The latter is given by

$$
\mathcal{A} \delta \sigma=\frac{1}{16 \pi G_{N}}\left(-2 a_{(d)}\right) \delta \sigma,
$$

since $\log \epsilon$ transforms with a shift whereas $a_{(d)}$ itself is invariant. Although this formula was derived under the assumption that $\delta \sigma$ is a constant, it follows from the general form of the conformal anomaly that it can be applied also for a non-constant $\delta \sigma$.

\section{The results}

The discussion in the preceding section shows that, up to a constant, the conformal anomaly of a theory related to a gravity theory only depends on the space-time dimension $d$. We will now evaluate the anomaly explicitly for the physically relevant cases $d=2,4,6$. 


\section{1 $d=2$ and the asymptotic symmetry algebra of $a d S_{3}$}

Here we get

$$
\mathcal{A}=-\frac{c}{24 \pi} R
$$

with the central charge

$$
c=\frac{3 l}{2 G_{N}} .
$$

This agrees with a computation based on the asymptotic symmetry algebra of three-dimensional anti-de Sitter space [10]!

\section{$3.2 d=4$ and $\mathcal{N}=4$ super Yang-Mills theory}

Here we get

$$
\mathcal{A}=-\frac{2 l^{3}}{16 \pi G_{N}}\left(-\frac{1}{8} R^{i j} R_{i j}+\frac{1}{24} R^{2}\right)
$$

Inserting for example the values of $l$ and $G_{N}$ appropriate for the $a d S_{5} \times S^{5}$ geometry of $N$ coincident $D 3$-branes in type IIB string theory, we get $N^{2}(\sim$ $\operatorname{dim} S U(N)$ ) times the conformal anomaly of an $\mathcal{N}=4$ supermultiplet (one vector, four chiral spinors and six scalars) [11]! The agreement between our strong coupling calculation and this weak coupling result indicates that there is a non-renormalization theorem for the conformal anomaly.

\section{3 $d=6$ and tensionless strings}

Here we get

$$
\begin{aligned}
\mathcal{A}= & -\frac{2 l^{5}}{16 \pi G_{N}}\left(-\frac{1}{128} R R^{i j} R_{i j}+\frac{3}{3200} R^{3}+\frac{1}{64} R^{i j} R^{k l} R_{i j k l}\right. \\
& \left.+\frac{1}{320} R^{i j} D_{i} D_{j} R-\frac{1}{128} R^{i j} D^{k} D_{k} R_{i j}+\frac{1}{1280} R D^{i} D_{i} R\right) .
\end{aligned}
$$

Inserting the values of $l$ and $G_{N}$ corresponding to the $a d S_{7} \times S^{4}$ geometry of $N$ coincident five-branes in $M$-theory, we find that $\mathcal{A}$ is proportional to $N^{3}$ (>> $\operatorname{dim} G L(N))$. This agrees with considerations based on the entropy of the brane system [12], [13]. This provides information about the mysterious tensionless string theory that appears when $M 5$-branes coincide. 


\section{A Appendix}

In this appendix we present some technical details of the calculation of the conformal anomaly.

Einstein's equation (3) for $G$ given in (4) amount to

$$
\begin{aligned}
\rho\left(2 g^{\prime \prime}-2 g^{\prime} g^{-1} g^{\prime}+\operatorname{Tr}\left(g^{-1} g^{\prime}\right) g^{\prime}\right)+l^{2} \operatorname{Ric}(g)-(d-2) g^{\prime}-\operatorname{Tr}\left(g^{-1} g^{\prime}\right) g & =0 \\
\left(g^{-1}\right)^{j k}\left(\nabla_{i} g_{j k}^{\prime}-\nabla_{k} g_{i j}^{\prime}\right) & =0 \\
\operatorname{Tr}\left(g^{-1} g^{\prime \prime}\right)-\frac{1}{2} \operatorname{Tr}\left(g^{-1} g^{\prime} g^{-1} g^{\prime}\right) & =0,(7)
\end{aligned}
$$

where differentiation with respect to $\rho$ is denoted with a prime, $\nabla_{i}$ is the covariant derivative constructed from the metric $g$ and $\operatorname{Ric}(g)$ is the $\operatorname{Ricci}_{\text {tensor }}{ }^{1}$ of $g$. We solve these equations iteratively in $\rho$.

The $g_{(k)}$ for $k<d$ in (5) are given by

$$
\begin{aligned}
g_{(2) i j}= & \frac{1}{d-2}\left(R_{i j}-\frac{1}{2(d-1)} R g_{(0) i j}\right) \\
g_{(4) i j}= & \frac{1}{d-4}\left(-\frac{1}{8(d-1)} D_{i} D_{j} R+\frac{1}{4(d-2)} D_{k} D^{k} R_{i j}\right. \\
& -\frac{1}{8(d-1)(d-2)} D_{k} D^{k} R g_{(0) i j}-\frac{1}{2(d-2)} R^{k l} R_{i k j l} \\
& +\frac{d-4}{2(d-2)^{2}} R_{i}^{k} R_{k j}+\frac{1}{(d-1)(d-2)^{2}} R R_{i j} \\
& \left.+\frac{1}{4(d-2)^{2}} R^{k l} R_{k l} g_{(0) i j}-\frac{3 d}{16(d-1)^{2}(d-2)^{2}} R^{2} g_{(0) i j}\right) .
\end{aligned}
$$

Although the above formulas for $g_{(k) i j}$ do not make sense for $d=k$, their traces are smooth in the $d$ goes to $k$ limit and in fact give the correct values for $\operatorname{Tr}\left(g_{(0)}^{-1} g_{(k)}\right)$ for $k \leq d$. Actually the easiest way to calculate the traces is to use the last equation in (7). By differentiating this equation with respect to $\rho$ and then setting $\rho$ to zero one obtains

$$
\begin{aligned}
& \operatorname{Tr}\left(g_{(0)}^{-1} g_{(4)}\right)=\frac{1}{4} \operatorname{Tr}\left(\left(g_{(0)}^{-1} g_{(2)}\right)^{2}\right) \\
& \operatorname{Tr}\left(g_{(0)}^{-1} g_{(6)}\right)=\frac{2}{3} \operatorname{Tr}\left(g_{(0)}^{-1} g_{(2)} g_{(0)}^{-1} g_{(4)}\right)-\frac{1}{6} \operatorname{Tr}\left(\left(g_{(0)}^{-1} g_{(2)}\right)^{3}\right)
\end{aligned}
$$

The above data are sufficient in order to obtain the anomaly up to $d=6$. In general, to determine the Weyl anomaly at dimension $d$ one needs to determine the full metric up to order $d-2$. The trace of the metric at order $d$ can be determined from metric at lower orders using the last equation in (7).

The explicit expression of $a_{(n)}$ 's in (6) can be derived by inserting the general form of the metric (4) in (1), perform the $\rho$ integration, and evaluate (2) at $\rho=\epsilon$. The coefficients $a_{(d)}$ (in $d$ dimensions) of the logarithmic divergences, which are

\footnotetext{
${ }^{1}$ Our conventions are as follows $R_{i j k}{ }^{l}=\partial_{i} \Gamma_{j k}{ }^{l}+\Gamma_{i p}{ }^{l} \Gamma_{j k}{ }^{p}-i \leftrightarrow j$ and $R_{i j}=R_{i k j}{ }^{k}$.
} 
relevant for the calculation of the Weyl anomaly, receive contributions only from the bulk integral. Their explicit form for $d=2,3,6$, are given by

$$
\begin{aligned}
a_{(2)}= & l \operatorname{Tr}\left(g_{(0)}^{-1} g_{(2)}\right) \\
a_{(4)}= & l^{3} \frac{1}{2}\left(\left[\operatorname{Tr}\left(g_{(0)}^{-1} g_{(2)}\right)\right]^{2}-\operatorname{Tr}\left[\left(g_{(0)}^{-1} g_{(2)}\right)^{2}\right]\right) \\
a_{(6)}= & l^{5}\left(\frac{1}{8}\left[\operatorname{Tr} g_{(0)}^{-1} g_{(2)}\right]^{3}-\frac{3}{8} \operatorname{Tr}\left[g_{(0)}^{-1} g_{(2)}\right] \operatorname{Tr}\left[\left(g_{(0)}^{-1} g_{(2)}\right)^{2}\right]\right. \\
& \left.+\frac{1}{2} \operatorname{Tr}\left[\left(g_{(0)}^{-1} g_{(2)}\right)^{3}\right]-\operatorname{Tr}\left[g_{(0)}^{-1} g_{(2)} g_{(0)}^{-1} g_{(4)}\right]\right)
\end{aligned}
$$

\section{References}

1. J.M. Maldacena, 'The large $N$ limit of Superconformal Field Theories and Supergravity', hep-th/9711200.

2. S.S. Gubser, I.R. Klebanov and A.M. Polyakov, 'Gauge Theory Correlators from Non-Critical String Theory', hep-th/9802109.

3. E. Witten, 'Anti-de Sitter space and Holography', hep-th/9802150.

4. C.R. Graham and J.M. Lee, 'Einstein Metrics with Prescribed Conformal Infinity on the Ball', Adv. Math. 87 (1991) 186.

5. L. Bonora, P. Pasti and M. Bregola, 'Weyl Cocycles', Class. Quant. Grav. 3 (1986) 635.

6. S. Deser and A. Schwimmer, 'Geometric Classification of Conformal Anomalies in Arbitrary Dimensions', Phys. Lett. B309 (1993) 279, hep-th/9302047.

7. M. Henningson and K. Skenderis, 'The Holographic Weyl Anomaly', JHEP 07 (1998) 023, hep-th/9806087.

8. G.W. Gibbons and S.W. Hawking, 'Action integrals and partition functions in quantum gravity', Phys. Rev. D15 (1977) 2752.

9. C. Fefferman and C.R. Graham, 'Conformal Invariants', in Elie Cartan et les Mathématiques d'aujourd'hui (Astérisque, 1985) 95.

10. J.D. Brown and M. Henneaux, 'Central charges in the canonical realization of asymptotic symmetries: An example from three-dimensional gravity', Commun. Math. Phys. 104 (1986) 207.

11. M.J. Duff, 'Twenty years of the Weyl anomaly', Class. Quant. Grav. 11 (1994) 1387, hep-th/9308075 and references therein.

12. S.S. Gubser and I.R. Klebanov, 'Absorption by Branes and Schwinger Terms in the World Volume Theory', Phys. Lett. B413 (1997) 41, hep-th/9708005.

13. I.R. Klebanov and A.A. Tseytlin, 'Entropy of Near-Extremal Black p-branes', Nucl. Phys. B475 (1996) 164, hep-th/9604089. 